\title{
ELM triggering by local pellet perturbations in type-I ELMy H-mode plasma at JET
}

\author{
P. T. Lang, B. Alper ${ }^{1}$, R. Buttery ${ }^{1}$, K. Gal ${ }^{2}$, J. Hobirk, \\ J. Neuhauser, M. Stamp ${ }^{1}$, and JET-EFDA contributors ${ }^{\dagger}$ \\ Max-Planck-Institut für Plasmaphysik, EURATOM Association, \\ Boltzmannstr. 2, 85748 Garching, Germany \\ ${ }^{1}$ EURATOM-UKAEA Fusion Association, Culham Science Centre, \\ OX14 3DB, Abingdon, OXON, United Kingdom \\ ${ }^{2}$ KFKI-RMKI, EURATOM Association, P.O. Box 49, H-1525 Budapest-114, Hungary \\ ${ }^{\dagger}$ See the Appendix of M. L. Watkins et al., Fusion Energy 2006 (Proc. 21st Int. Conf. Chengdu) IAEA (2006)
}

\begin{abstract}
The pellet pacing ELM control concept developed at the mid sized tokamak ASDEX Upgrade is considered for the larger machines JET and ITER as well. By driving up the ELM frequency, the ELM size can be reduced and eventually suppressed below a dangerous level. An according pellet injection systems for JET is under construction, the ITER design includes one as well. However, it has still to be proven the concept will work also at bigger machine sizes. A step forward in this mission was achieved by re-analysing previous JET experiments dedicated to pellet particle fuelling. Although the experiments were performed in a parameter regime unsuitable for pellet ELM pacing both with respect to pellet frequency and size they demonstrate prompt triggering of ELMs takes place at JET as well. Prompt triggering, an indispensable premise for useful pacing, obviously can be achieved already with a pellet size sufficiently small to avoid unbearable side- effects by particle fuelling. An ELM released by the local perturbation imposed by the ablating pellet in the plasma edge region is detected when only a minor fraction (less than $1 \%$ ) of the fuelling size pellets (containing about $4 \times 10^{21} D$ ) was ablated and deposited yet. It is thus very likely JET will allow for investigations on the operational technique and the underlying physics of pellet pacing once the new system becomes operational.
\end{abstract}




\section{Introduction}

While developing reactor relevant plasma scenarios en route to ITER edge localised modes (ELMs) occurring in high confinement mode (H-mode) discharges turned out to form a considerable hassle. With growing tokamak size they become a significant issue requiring control efforts. It was found ELMs cause a burst-like energy transport out of the plasma resulting in transient heat pulses onto in-vessel components. Type-I ELMs characteristic for typical standard scenarios show a tendency towards less frequent appearance with growing machine size. Since the energy fraction expelled by ELMs stays approximately constant in a wide parameter regime [1] resulting peak power loads drastically increase. Plasma facing components exposed to the most intense part of the ELM induced pressure pulse, usually the divertor plates, can be stressed to their limits and even beyond. Up to now for mid-size tokamaks like ASDEX Upgrade type-I ELMs did not cause major concerns. However, in todays largest machines like JET their destruction potential is already perceptible in some high performance discharges [2]. For ITER fatally fast erosion in the divertor region will be caused without control [3].

Several approaches to solve this problem are currently under investigation. For example the reduction of the energy flux to the wall by establishing a radiative edge $[4,5]$ or by suppressing ELMs at all by edge ergodisation [6] was demonstrated. Another option is to establish ELM control by the pacing concept. Here the approach aims on forcing ELMs to appear on a frequency different from the unperturbed frequency of spontaneous ELMs $f_{E L M}^{0}$ by manipulations in the edge plasma. The basic idea is to raise the controlled ELM frequency $f_{E L M}$ above $f_{E L M}^{0}$ and accordingly reduce the energy per ELM, if required below the required danger level. Beneficial increase of $f_{E L M}$ can be achieved by different means, the injection of cryogenic solid pellet produced from hydrogen fuel so far found the most successful. Experiments performed at ASDEX Upgrade [7] demonstrated full ELM control once the pacing frequency given by the pellet injection rate $f_{P e l}$ yields $f_{P e l}>1.5 \times f_{E L M}^{0}$. Once ELM control is achieved only triggered ELMs appear anymore with a frequency which equals the pellet frequency $f_{P e l}=f_{E L M}$. It also turned out pellet driven type-I ELMs preserve the typical ELM characteristics and appear essentially identical compared to spontaneous ELMs with the same frequency. After this proof-of-principle the concept of ELM mitigation via pellet pacing was included into the ASDEX Upgrade standard tool kit of control features and applied on several occasions [8]. Pellet pacing systems have been developed or are under development for tokamak devices like DIII-D [9] and JET [10] and is integrated into the ITER concept [11] as well. However, as the basic physics of the ELM triggering process is yet unknown the efficacy of pellet pacing in larger machines is not guaranteed. Hence, investigations in larger sized machines are especially valuable to clarify the situation.

We made use of the stepladder AUG JET ITER to approach ITER size by investigating pellet ELM triggering at JET. An according analysis performed at JET will be presented in the following. For performing a full mitigation experiment JET however lacks a suitable injection system yet.

Previous experiments dedicated to particle fuelling studies and high density operation applying pellet injection had been rather far from ideal pacing conditions. Their pellet frequency usually was too low while the pellet size was much too big. Large pellet masses adapted for the fuelling purpose altered drastically also the plasma parameters during a pellet sequence. Hence, the full mitigation scenario cannot be studied under such conditions. Nevertheless, even in such experiments the initial ELM trigger process initiated by the pellet can be studied. The possibility to gain useful information on the physics of the ELM triggering even under circumstances far from pacing pa- 
rameters was recently demonstrated [12]. There, a low pellet rate $f_{P e l}<<\times f_{E L M}^{0}$ was applied to avoid persistent perturbation of the target plasma while investigating local conditions of the ELM onset.

In a similar way the analysis presented in the following concentrates on the question if and how ELM triggering is achieved at the larger size of JET by pellet injection into type-I ELMy H-mode phases. Detailed investigations of the question weather the ELM can be released already by the direct local 3D perturbation created by the ablation cloud in the edge barrier region are presented. Only if prompt triggering can be achieved by injection of small pellets avoiding high fuelling rates pellet pacing will become a useful control at JET as well. In case ELM triggering requires the global impact of a large pellet on a time scale typical for radial particle and energy transport, the resulting fuelling would cause a change of plasma parameters making ELM control efforts invalid. In the following we will show prompt ELM triggering is achieved with pellet injection at JET and thus this essential prerequisite for envisaged pacing attempts is fulfilled.

\section{Set up}

The JET centrifuge based pellet system was operated from 1995 until 2003, and was able to inject pellets at a size adapted for fuelling requirements ( $4 \mathrm{~mm}$ cubes, nominal $\left.3.8 \times 10^{21} \mathrm{D}\right)$ at shot-to-shot variable speeds in the range 150 to $300 \mathrm{~m} / \mathrm{s}$. In typical fuelling scenarios these pellets penetrate about to $\rho=0.7-0.8$ enhancing the plasma particle inventory by about $10-30 \%$. In its final stage, pellet launch was possible from three different poloidal positions through separate guiding tracks [2]. The three designated injection trajectories are shown in figure 1, showing the poloidal cross-section of JET at octant 2. A track selector unit was installed at the exit of the centrifuge. It could switch sufficiently fast to direct any single pellet to either one of the three tracks or to suppress its injection by guiding it to a pellet dump.

The injection tracks are referred to as the inboard or high field side $(\mathrm{H})$, outboard or low field side (L) and top or vertical (V) track. In addition, the observation cone of the pellet monitor and the area covered by the vertical soft X-ray (SXR) cameras [13] 35 lines of sight are displayed. $D_{\alpha}$ radiation mainly from the ablation zone optically accessed through a telescope and a quartz fibre was recorded by fast photomultipliers. This signal can be used as a fairly good measure of the pellet ablation rate $\frac{d N_{P}}{d t}$ [14] however taking into account viewing radiation from regions close to the first wall can create features not stemming from the ablation process. While the telescope's view covers the initial part of the $\mathrm{H}$ track in the plasma, it misses the ablation onset for $\mathrm{V}$ and $\mathrm{L}$ pellets, the latter due to a slight toroidal displacement.

Measurements of electron density and temperature by a DCN interferometer and an ECE radiometer provide sufficient temporal resolution for a clear detection of the pellet impact. However, since rapid and pronounced local pellet particle deposition causes fringe phase loss and density cut-off, the absolute values measured become perturbed and data can be used for qualitative analysis only. Onset of strong burst like MHD activity is a clear signature of the ELM providing a reliable and fast onset marker. Mirnov coils mounted at different toroidal and poloidal locations were employed for their detection. Observing $D_{\alpha}$ radiation from several lines of sight covering e.g. divertor strike point regions was used for ELM monitoring, too. Sensitive to the ELM induced pressure pulse arriving at approximately ion sound speed they are considerably slower than magnetic signals but valuable for confirmation and validation of the Mirnov monitor. 


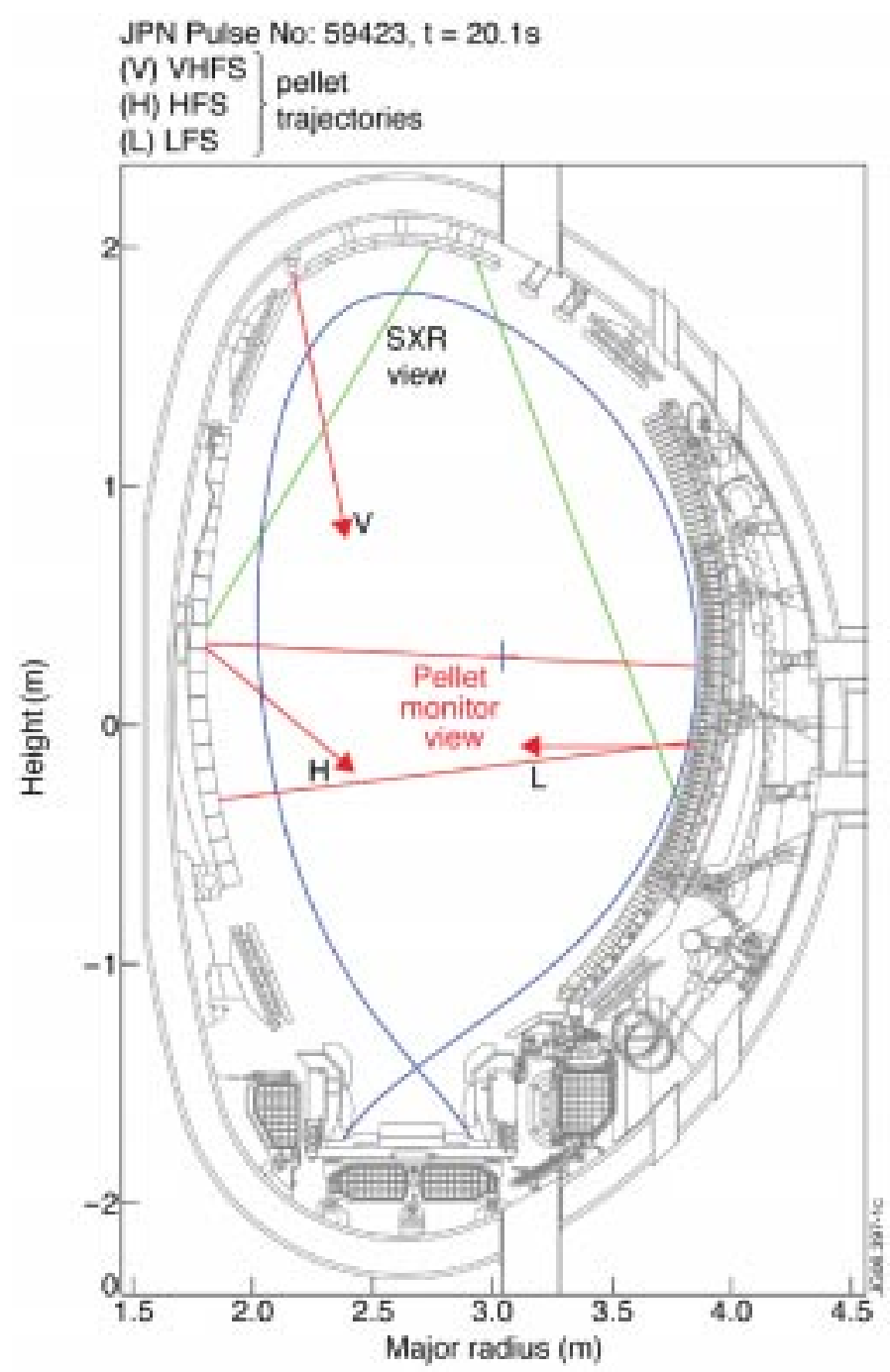

Figure 1: Poloidal cross section of JET octant 2 with a typically used plasma separatrix contour, the three designated pellet injection tracks, observation areas covered by SXR camera and pellet ablation monitor.

\section{Analysis}

\subsection{Experimental conditions}

Experiments analyzed here were originally performed to compare the three launch positions with respect to their fuelling performance. Pellet injection at $f_{P e l}=6 \mathrm{~Hz}$ took place in short sequences from different locations separated by time gaps so that the plasma could return to its initial conditions. A plasma configuration referred to as HT (high triangularity) scenario, proven to be already suitable for pellet fuelling, had been chosen [15]. With a total heating power of about 18.5 MW (15 MW NBI, about $3 \mathrm{MW} \mathrm{RF)} \mathrm{the} \mathrm{plasma} \mathrm{was} \mathrm{driven} \mathrm{well} \mathrm{into} \mathrm{the} \mathrm{H-mode} \mathrm{regime}$ (threshold about $8 \mathrm{MW}$ ). Initial plasmas typically developed an ELM-free phase lasting for about $100 \mathrm{~ms}$ followed by sequences of clear type-I ELMs at about $50 \mathrm{~Hz}$ of similar duration. The first ELM of the sequence usually displayed the largest amplitudes in the ELM monitors about 2 - 4 times higher than the remaining ELMs in the 
train. Pellet fuelling transiently modified this behaviour naturally towards a more uniform ELM pattern at slightly higher frequencies. Nevertheless, from an ELM pacing point of view these conditions (at least for the first few pellets in the train at the onset of pellet ablation) can be considered close to the case refereed to as perturbative mode $[12,16]$.

\subsection{Data selection strategy}

Although every single pellet injected proved to be a suitable event to study its impact on ELM behaviour, special care has to be taken in order to achieve validated data. Only data with sufficient temporal resolution are selected for a reasonable analysis. In order to fulfil the first requirements we selected only cases where the pellet track could be definitely determined by its time-of-flight and the pellet arrived in obviously good shape (with exception of the sole L event picked). To meet the second selection criterion, in the database we included only pellets having their data recorded as well during a time window of the fast data acquisition system (CATS) which runs at $250 \mathrm{kHz}$ sampling rate. Altogether, we found $5 \mathrm{H}($ all $150 \mathrm{~m} / \mathrm{s}), 10 \mathrm{~V}(7$ at $150 \mathrm{~m} / \mathrm{s}, 3$ at $240 \mathrm{~m} / \mathrm{s})$ and $1 \mathrm{~L}(240 \mathrm{~m} / \mathrm{s})$ pellets.

\subsection{Experimental results}

\subsubsection{Reliable ELM triggering by pellets}

Beside the well characterized pellets many others not included in the database were found to trigger an ELM during the lifetime of the pellet. This confirms and broadens earlier findings in hot-ion discharges [17]. In particular, it turns out an ELM can be triggered any time, i.e. at any stage of the intrinsic ELM cycle or during the ELM free phases. An example is shown in figure 2 where we compare in the upper part several intrinsic and two triggered ELMs. One is triggered shortly after (about $8.5 \mathrm{~ms}$ ) an event in the phase of declining ELMs (using the outer divertor $D_{\alpha}$ monitor signal) during the intrinsic ELM train, the other after an ELM-free phase lasting $87 \mathrm{~ms}$.

Besides the fact that pellets trigger ELMs at a time intrinsic ones are unlikely to appear it is also obvious the pellets alter significantly the ELM behaviour while the fuelling impact (visualized by density and temperature evolution) lingers on. Details on an enhanced time scale as recorded by the CATS system for both hatched sequences are plotted in the lower part of figure 2. The ELM triggered $87 \mathrm{~ms}$ after its intrinsic predecessor (right) is a strong one as typical for leading events in the sequence. A cascade of ELMs intensified in comparison to the earlier intrinsic train onset follows. This is likely due to strong fuelling resulting in a massive out flux of particles, documented as typical behaviour for the injection of large pellets in ASDEX Upgrade [7]. The ELM triggered shortly after an intrinsic one (right) turns out less pronounced but is followed by a fuelling-cascade as well.

\subsubsection{Prompt ELM triggering by pellets}

Both triggered ELMs displayed in figure 2 show indications that they consist of two components. The first is hefty and short, the second gradual and persistent. The first component is the desired promptly triggered ELM induced by the local pellet perturbation in the edge region, as will be shown in the following. The second can be a strong limitation for the pellet pacing approach and should therefore be avoided or at least minimized by choosing pellet parameters restricting unwanted fuelling to the lowest possible amount. The decisive questions and hence subject 

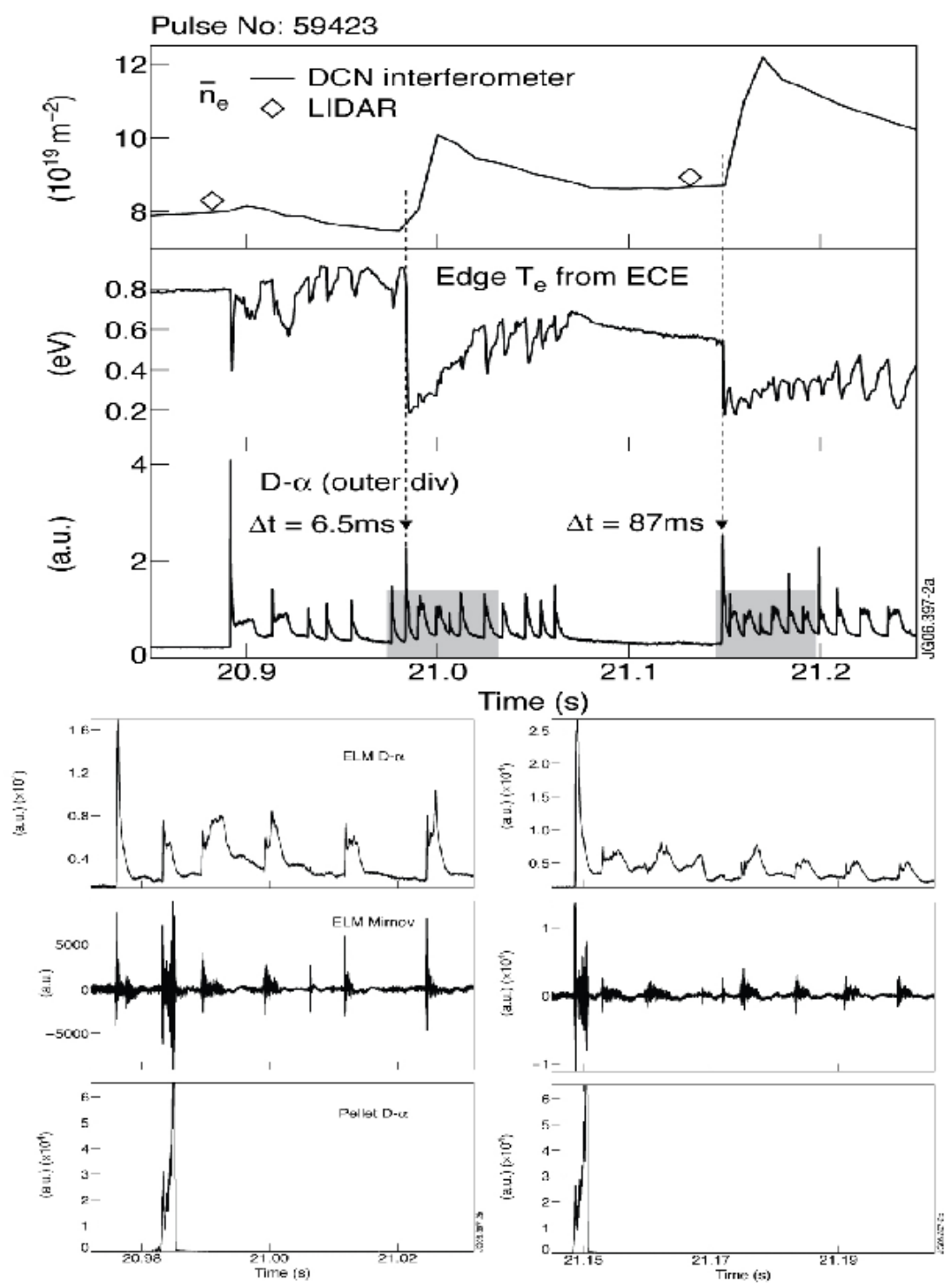

Figure 2: Pellets triggering ELMs at arbitrary times in the ELM cycle. Upper part (from top): electron density - line averaged from DCN interferometer (solid line) and central value from LIDAR system (open diamonds); electron temperature at the edge from ECE, due to signal cut-off caused by the transiently enhanced edge density after pellet particle deposition the measured temperature drop just after pellet injection is most probably exaggerative; outer divertor $D_{\alpha}$ ELM monitor. Both pellets trigger an ELM despite a different elapsed time with respect to the previous event. The two hatched sequences are displayed on an expanded time scale in the lower part. Lower part signals recorded by the high temporal resolution CATS system (from top): $D_{\alpha}$ and MHD ELM monitors, ablation monitor signals. Pellet induced ELMs clearly consist of a fast and a slower component, the latter thought to be fuelling induced.

of our detailed investigation is now: when and where does the pellet trigger this prompt ELM and what would be as a consequence the minimum required size for pacing pellets. It is ultimately this value that determines the usefulness of the pellet pacing tool. 

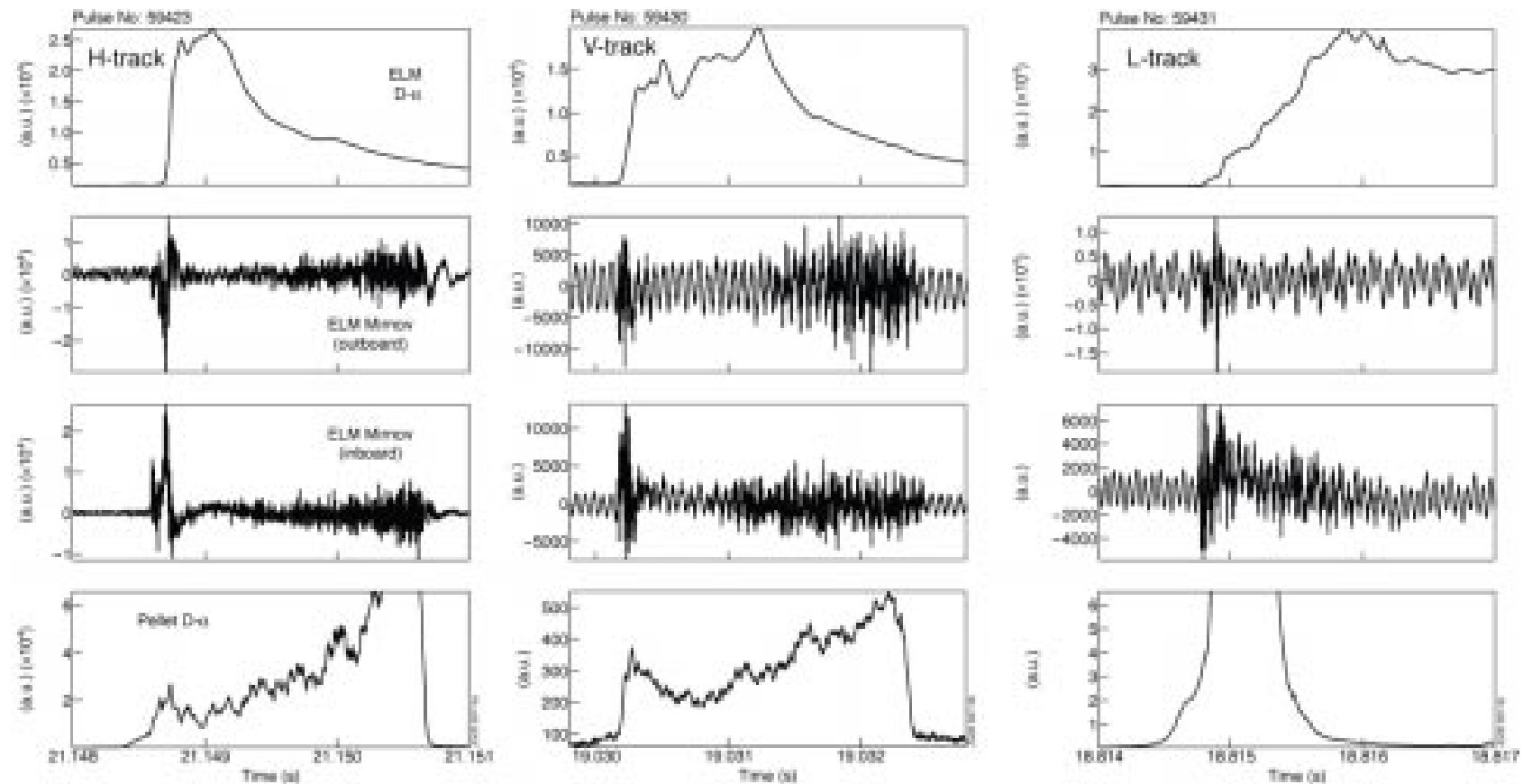

Figure 3: $D_{\alpha}$ and MHD (outboard and inboard Mirnov coils) ELM monitors, pellet monitor (top to bottom) for pellet launch from inboard/high-field-side (H), top/vertical (V) and outboard/low-field-side (L)track (left to right).

In figure 3, observed pellet ablation and resulting ELM behaviour is shown for all of the three different injection locations. On the time scale used for the plot (every box covers $3 \mathrm{~ms}$ ) the first strong ELM component can clearly be seen starting very early in the ablation process when little pellet mass has been ablated yet. For $\mathrm{H}$ and V launch, where typical examples were chosen, it can also be seen that the fuelling induced component, mainly recognized by the elevated MHD activity, lasts longer than intrinsic and promptly triggered events. This fuelling driven component takes place on a much longer timescale than the prompt one, in a radial transport dominated temporal regime. This should be avoidable in case sufficiently early pellet burn out can be achieved. The single outboard case undoubtedly attributed to a pellet launch shows the same tendency but with less clearly separated phases. However, the ablation monitor signal displays behaviour rather not typical for pure pellet ablation. The belief is that the pellet has disintegrated and what is arriving here is a composite of pellet debris and gas. Obviously, a technical problem with the track selector in the L position damaged or even destroyed pellets yet before entering the $\mathrm{L}$ track. This conclusion is supported by the small impact on density and temperature by this pellet in particular and the low delivery reliability of pellets when launched via the L track in general. Nevertheless, even this pellet debris seems already sufficient to create a prompt ELM.

The pellet ablation rate and hence the ablation monitor signal depends - for fixed pellet parameters - mainly on density and temperature of the target plasma. Under H-mode conditions with its pronounced density and temperature gradients at the edge, the onset of detectable ablation radiation (here typically $1 \%$ of its peak value at pellet burn out) is correlated well with the pellet passing the separatrix as found by recent pellet tracking experiments employing fast framing camera systems [12]. As mentioned earlier, this ablation onset is visible for the $\mathrm{H}$ track only. Hence, determination of the prompt ELM onset and estimation for the minimum pellet mass ablation at this 
time can be performed only for this case. Although the comparison of the three tracks does not indicate a drastic difference, a more detailed investigation of the influence of the poloidal pellet position on the ELM onset is still required once better-adapted diagnostics are at hand. The analysis of the ELM onset requirements is, as it represents a typical example, performed for the $\mathrm{H}$ track pellet shown in figure 4. For comparison, an intrinsic ELM as recorded in a reference discharge is plotted as well.

This comparison again clearly shows the similarity of the ELM fingerprints in the recorded monitor signal for an intrinsic and a promptly triggered ELM. To find out the required residual unavoidable fuelling burden for ELM pacing, the optimised pellet parameters mass $m_{P}$ and velocity $v_{P}$ has to be determined. For the best choice a pellet of minimum mass must still provide a sufficient perturbation to trigger prompt ELMs. As the trigger mechanism is yet unknown and an analysis of the delay between imposing the perturbation and the onset of the ELM lacks appropriate data [12], we determine for this given plasma and pellet parameters the ablated particle amount and penetration depths at the moment of the observed ELM onset. The ablation monitor signal recorded for the intrinsic ELM reveals there is radiation arising from the ELM. This additional component is superposed on the gradually increasing ablation radiation signal, causing an additional hump on it. The kink in the ablation monitor signal resulting from the sharp onset of this hump correlates also with the ELM onset in the MHD monitor signal and is therefore taken as the ELM onset. Eliminating direct ELM radiation and assuming the remaining $D_{\alpha}$ radiation is proportional to the ablation rate, it is calculated the pellet lost only about $1 \%$ of its initial mass at ELM onset.

By attributing detectable ablation onset to the pellet's crossing the separatrix, a penetration depth $\Delta s$ of about 30 $\mathrm{mm}$ along the designated path is obtained. With the $\mathrm{H}$ track tilted by 40 degrees with respect to the horizontal, the pellet is by then only about $\Delta r=20 \mathrm{~mm}$ inside the separatrix. For all $\mathrm{H}$ track pellets in the database, an ELM onset delay of $200 \pm 30 \mu s$ is derived, related to $\Delta s=30 \pm 4.5 \mathrm{~mm}$ or $\Delta r=23 \pm 3.4 \mathrm{~mm}$, respectively.

\subsubsection{Comparison of trigger, ELM and pellet perturbations}

The analysis confirms prompt ELM triggering first observed at ASDEX Upgrade is achievable as well at the larger size of JET. Triggered ELMs show, at least from data available so far and before fuelling effects become dominant, no significant difference with respect to their intrinsic counterparts. Consumption of only a minor amount of a fuelling sized pellet is sufficient for releasing the ELM. This might hint for a possibility on a reduction of the applied pellet size and hence the fuelling burden. However, to map out precisely operational plasma and pellet parameter conditions under which pacing can be still maintained require more dedicated investigations on this issue with a better adapted injection system.

An illustration of the prompt ELM being triggered without significant changes of the local plasma density and temperature is presented in figure 5. It shows the evolution of the SXR emission profile, displayed by equal intensity contour lines, for an intrinsic ELM (upper) and a pellet injection event (lower). For the triggered ELM it can be seen pellet ablation monitored by the $D_{\alpha}$ radiation has just set in when the ELMs MHD activity becomes visible in the Mirnov signal monitor. With the ELM evolving, increasing energy out flux and particle recycling at the wall causes some additional $D_{\alpha}$ radiation from the observation area. This creates the small hump for the intrinsic reference case and the larger extra hump in the pellet triggered case. The larger amount of additional $D_{\alpha}$ radiation created by the ELM superimposed on the ablation component is attributed to a higher particle losses from 

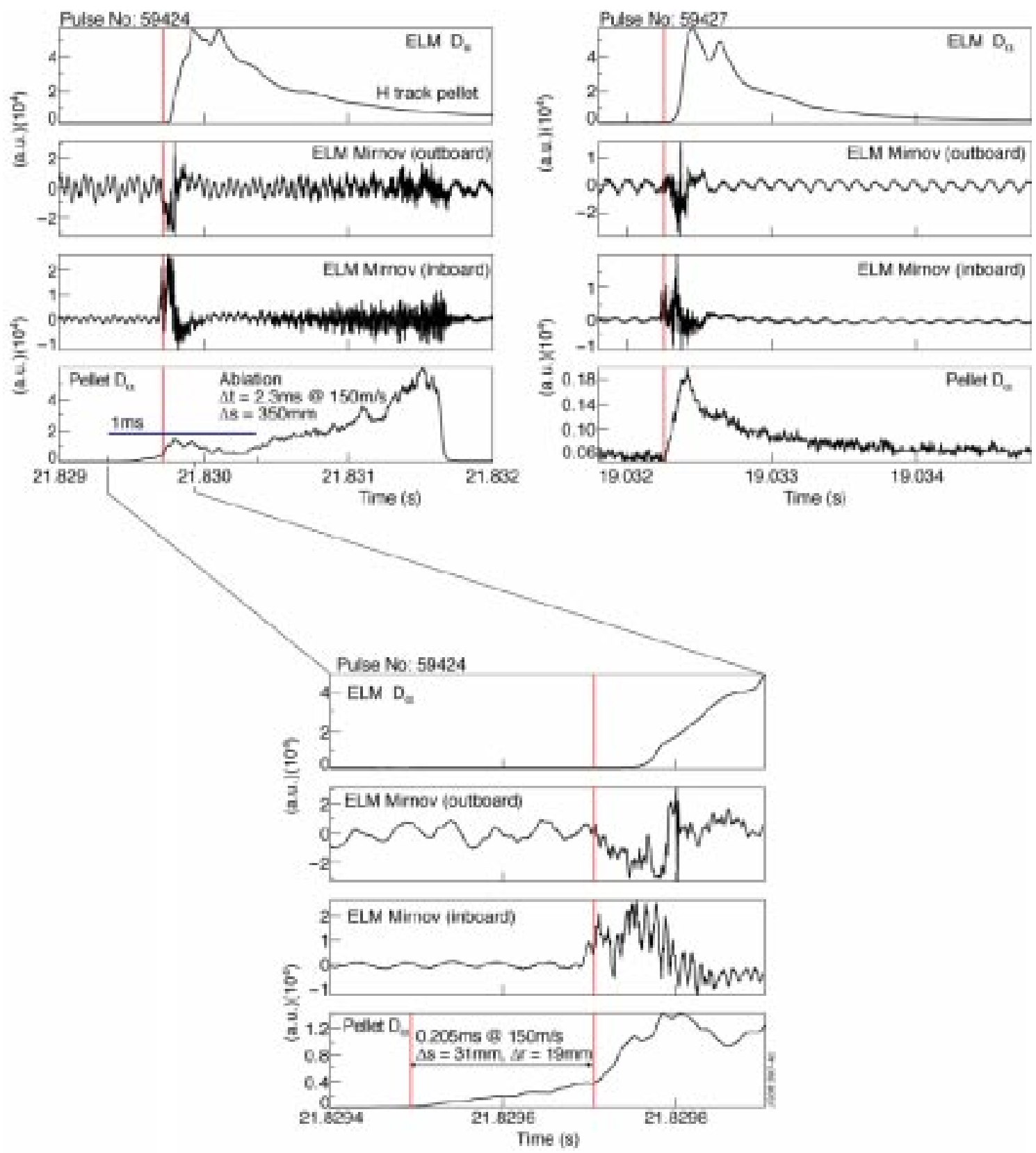

Figure 4: Upper: Comparison of triggered (left) and intrinsic (right) ELM. Signals from top: $D_{\alpha}$ outer divertor, MHD outboard and inboard ELM monitors, ablation monitor. Lower: expansion of ablation and ELM onset for triggered ELM.

the according edge region. This is due to the local density enhancement quite far beyond the equilibrium level by the pellet particle deposition in this area.

The observed SXR emissivity change is mainly attributed to a reduction of the local temperature. A pellet induced cooling effect can just be identified when the triggered ELM and its resulting edge cooling sets in. The ELM then dominates the edge region for about $0.2 \mathrm{~ms}$ until the pellet impact gradually becomes dominant again. When the 


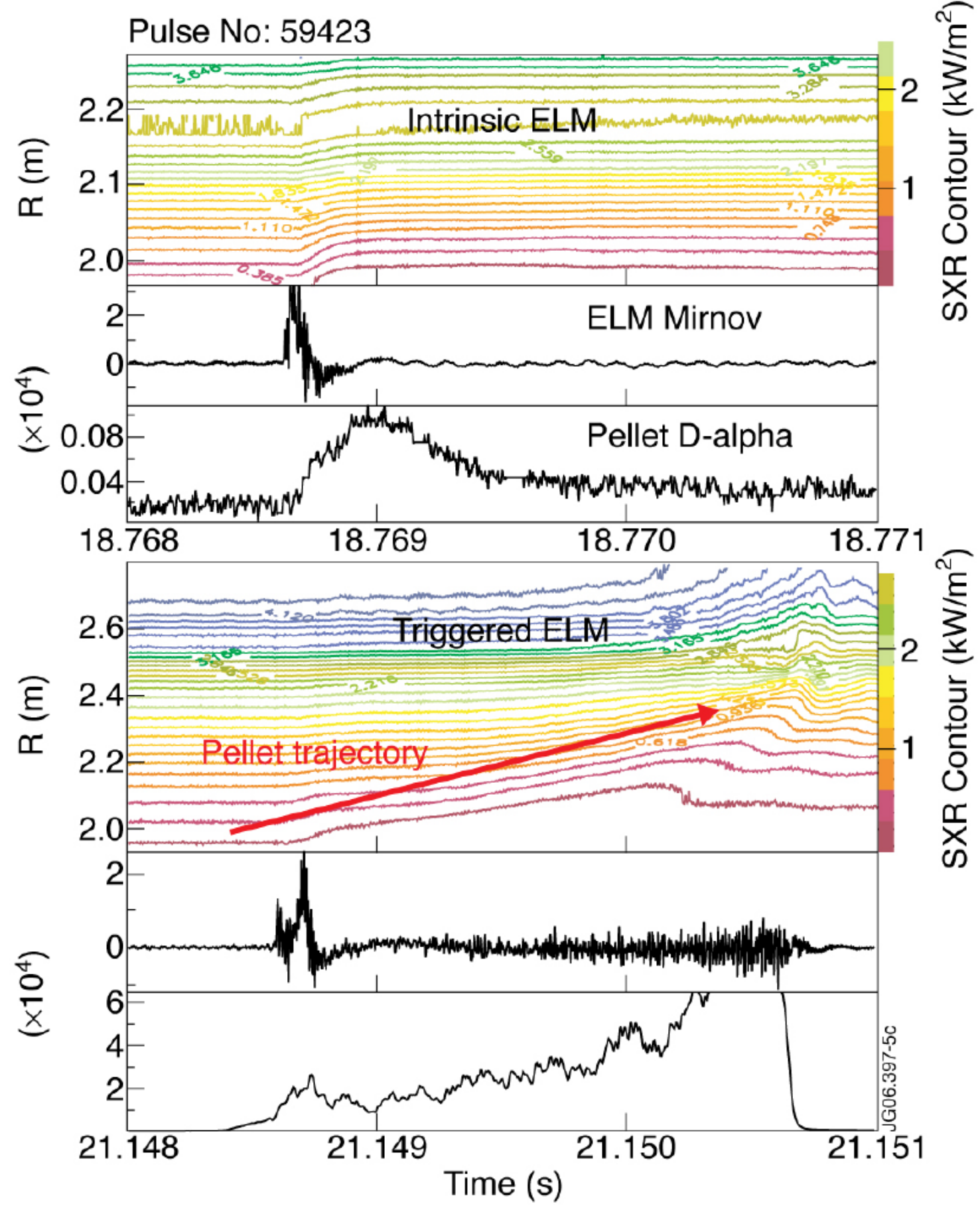

Figure 5: Spatio-temporal evolution of SXR equivalence contour lines in the case of an intrinsic ELM (upper) and a pellet triggered ELM (lower). The emissivity amplitude is colour coded, scale shown at the right side. For the triggered case, the pellet motion along its designated trajectory is mapped into the SXR pattern. As well, ELM Mirnov monitor and the pellet ablation signals are plotted showing the temporal evolution of pellet ablation and ELM onset. 
pellet is burnt out, its final local cooling effect in the edge region exceeds that one of a typical ELM by about a factor of 10 and that one imposed by the pellet in the moment the trigger ELM starts by about a factor of 100. Here again it becomes clear the pellet impact required for ELM pacing is small compared to the ELM impact itself but a fuelling sized pellet in turn creates a much larger perturbation potentially spoiling any advantage from pacing.

\section{Enhancement potential by mass adaptation}

Although this experiments using large pellets demonstrated a prompt ELM can be triggered by depositing only about $4 \times 10^{19} \mathrm{D}$ in the plasma edge, it is not self-evident that just a drastic reduction in pellet mass is required to achieve almost fuelling free pacing since there is also feedback on the ablation characteristics. Anyhow a reduction of pellet mass causes a reduction of the pellet ablation rate $\frac{d N_{P}}{d t}$, which may reduce the perturbation below the triggering threshold. As the ablation rate depends on the pellet mass as $\frac{d N_{P}}{d t} \sim m_{P}^{4 / 9}[18,19]$, reducing the pellet mass to 0.01 times the initial value would cause a reduction to about 0.1 times the initial ablation rate. So far all investigated pellet injection events into type-I ELM phases of AUG and JET plasmas were found to cause a prompt ELM trigger. This might indicate the pellet perturbation created is significantly beyond a required trigger threshold. Pellets may perturb the edge by several mechanisms, like density perturbation or cooling etc. Regarding the density perturbation it is still headroom for a reduction of pellet mass $m_{P}$. In this case the perturbation is imposed by particle deposition along the pellet path it is proportional to $\frac{d N_{P}}{d r}=\frac{\frac{d N_{P}}{d r}}{v_{P}^{r}}$ with $v_{P}^{r}$ the radial pellet velocity. Reducing the pellet mass the resulting ablation rate may become too small for sustaining prompt ELM triggering at the initial pellet velocity. Assuming that the ablation rate has a week dependence on the pellet velocity, the recovery of the perturbation strength inversely proportional with the pellet velocity reduction may be expected. By velocity reduction the pellet ablation is more concentrated over the edge region, increasing its perturbation strength with respect to the ELM. Reduction of $v_{P}^{r}$ can be achieved by reducing the launch velocity and an increase of the trajectory inclination angle. Technically, a reduction of the actual launch velocity to about $60 \mathrm{~m} / \mathrm{s}$ is reasonable with simple measures; a re-design of the injection path however is a major effort.

To get a first quantitative indication on the influence of pellet size and velocity on the relevant radial particle deposition rates under actual JET conditions, a modelling attempt was made. The simulations were made by the hybrid code which [20] treats both the spherically expanding neutral and the field elongated ionized cloud. Taking the pellet shown in figure 4 and the plasma electron density and temperature profiles just before injection as reference case, pellet particle deposition rates were computed for different pellet sizes and velocities. Results are shown in figure 6, displaying obtained $\frac{d N_{P}}{d r}$ values versus major plasma radius for the reference case and two comparison cases. For the code runs, spherical pellets with radii adapted to match the real $m_{P}$ values were considered and the effective speed $v_{P}^{r}$ was adopted as well. Reducing the pellet mass to $1 \%$ of the reference value while keeping $v_{P}^{r}$ constant reduces the resulting deposition rate by a factor of about 10. As a consequence of the reduced ablation rate, the smaller pellet passes well beyond the location of the reference pellet at the moment when the ELM onset is observed. Due to the pellet produced perturbation scaling very roughly as $\frac{\sqrt{m_{P}}}{v_{P}}$, a speed reduction by a factor of 10 would be expected able to restore the reference case. However, code calculations indicate for an exhaust of the ablation potential for very low pellet speed. A similar effect was already reported earlier [21]. This effect needs further considerations for its importance of the potential for deposition recovery by velocity reduction. 
To visualize the velocity induced deposition recovery an example is shown as well in figure 6 with again $1 \%$ reference $m_{P}$ value but at a reduced injection velocity. The reduction in $v_{P}$ elevates the deposition rate recovering the reference value to a large extent.

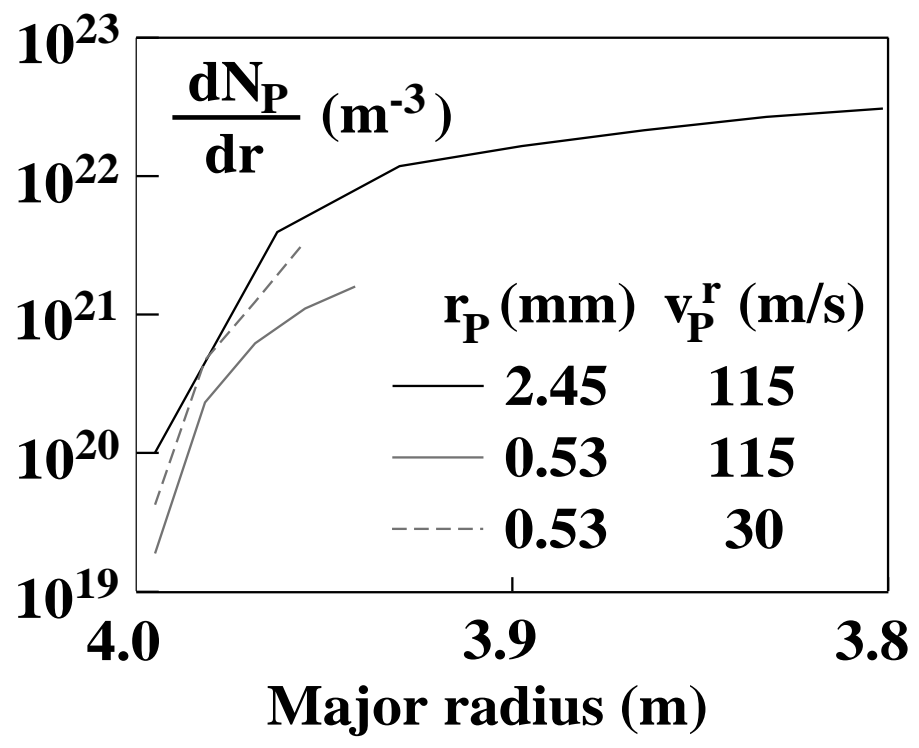

Figure 6: Pellets deposition rates calculated for the pellet shown in figure 4 as reference case (black solid). Reduction of the pellet mass to about $1 \%$ of this reference value (indicated pellet radii refer to spherical pellets of equivalent mass to real cubes) reduces the deposition rate by a factor of about 10 (solid grey). Reducing the pellet speed as well can recover towards the reference deposition profile (dashed grey).

To find out to which extend a pellet size reduction can be achieved while keeping the trigger potential require further enhanced modelling. It is requested to obtain precise knowledge on size, structure and amplitude of the pellet produced perturbation. On the other hand, more information on the threshold conditions for a pellet produced perturbation to an ELM are required. As already mentioned above, to gain this information detailed experimental investigations performing pellet mass and velocity scans by varying the imposed perturbation in the pedestal are required.

\section{Summary and outlook}

Pellet injection into JET type-I or ELM free phases was found able to trigger an ELM at any time. Detailed investigations of the triggered ELM onset dynamics in comparison with the ablation evolution demonstrated prompt triggering relying on a sufficiently strong local perturbation in the edge - shown before only in mid-size experiments - does still work on a JET-size machine. Thus, the ELM pacing approach seems to have a good prospect for application even at reactor scales. Pellets with a particle content of only about $4 \times 10^{19} \mathrm{D}$ could be sufficient for ELM pacing in JET but eventually require a reduced radial velocity to compensate for the reduction of ablation rate with pellet size. Hence, the particle flux resulting can possibly be suppressed to negligible amounts eliminating fuelling constraints which hamper current investigations. 
A new pellet injection system has been designed and built for JET which will provide in addition pellet parameters optimized for pacing requirements [10]. It is expected to become operational by late 2007. This new injector, together with pellet launch options from different poloidal positions and powerful diagnostic equipment [2] will make JET a most appropriate facility for further pacing investigation. 


\section{References}

[1] HERRMANN, A., Plasma Phys. Controlled Fusion 44 (2002) 883.

[2] PAMELA, J. et al., The contribution of JET to ITER, in 24th SOFT Conference, Warsaw, 2006, pages PL1-O-551, 2006.

[3] FEDERICI, F. et al., Plasma Phys. Controlled Fusion 45 (2003) 1523.

[4] KAlLENBACH, A. et al., Plasma Phys. Controlled Fusion 38 (1996) 2097.

[5] RAPP, J. et al., Nucl. Fusion 44 (2004) 312.

[6] MOYER, R. A. et al., Phys. Plasmas 12 (2005) 056119.

[7] LANG, P. T. et al., Nucl. Fusion 43 (2003) 1110.

[8] HORTON, L. D. et al., Plasma Phys. Controlled Fusion 46 (2004) B511.

[9] BAYLOR, L. et al., Bull. Am. Phys. Soc. 51, 115 (2006) .

[10] GERAUD, A., The JET high frequency pellet injector project, in 24th SOFT Conference, Warsaw, 2006, pages P4-H-201, 2006.

[11] POLEVOI, A. et al., Nucl. Fusion 43 (2003) 1072.

[12] KOCSIS, G. et al., submitted to Nucl. Fusion .

[13] ALPER, B. et al., Rev. Sci. Instrum. 68 (1997) 778.

[14] MC NEILL, D., J. Nucl. Mater. 162-164 (1989) 476.

[15] LANG, P. T. et al., Nucl. Fusion 42 (2002) 388.

[16] LANG, P. T. et al., Plasma Phys. Controlled Fusion 48 (2006) A141.

[17] ALPER, B. et al., ELMs, precursors and triggers on JET, in Europhysics Conference Abstracts (CD-ROM, Proc. of the 29th EPS Conference on Plasma Physics and Controlled Fusion, Montreux, 2002), edited by BEHN, R. and VARANDAS, C., volume 26B, page P1.025, Geneva, 2002, EPS.

[18] PARKS, P. B. and TURNBULL, R. J., Phys. Fluids 21 (1978) 1735.

[19] PARKS, P. B. and ROSENBLUTH, M. N., Phys. Plasmas 5 (1998) 1380.

[20] GÁL, K. et al., 31 ${ }^{\text {nd }}$ EPS Conf., London 2004, P5.149 .

[21] NEUHAUSER, J. and WUNDERLICH, R., A model for periodic pellet ablation in toroidal plasma confinement experiments, Technical Report 5/30, IPP, Garching, Germany, 1989. 